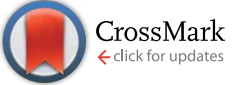

Cite this: RSC Adv., 2015, 5, 63615

\title{
Transfer of SABRE-derived hyperpolarization to spin-1/2 heteronuclei $\dagger$
}

\author{
Andrey N. Pravdivtsev, ab Alexandra V. Yurkovskaya, ${ }^{\text {ab }}$ Herbert Zimmermann, ${ }^{c}$ \\ Hans-Martin Vieth ${ }^{d}$ and Konstantin L. Ivanov ${ }^{\star a b}$
}

In this paper, we describe a method of hyper-polarizing "insensitive" Nuclear Magnetic Resonance (NMR) nuclei by exploiting the SABRE (Signal Amplification By Reversible Exchange) technique and transferring spin order from protons originating from parahydrogen. We demonstrate that hyperpolarization transfer is due to a coherent mechanism, which is operative at (i) very low magnetic field; (ii) geomagnetic field; (iii) high field in the presence of a suitable radiofrequency-excitation scheme. Experiments are performed using ${ }^{15} \mathrm{~N}$-labelled pyridine as the SABRE substrate; NMR enhancements achieved for ${ }^{15} \mathrm{~N}$ nuclei are more than 1000 for free pyridine in solution and more than 20000 for pyridine bound to the SABRE complex. High-field SABRE experiments are particularly important for enhancing the sensitivity of NMR methods: they enable strong signal enhancements and avoid technically demanding field-cycling. Furthermore, such experiments use very low power for NMR excitation and make feasible continuous re-hyperpolarization of the substrate in high-field experiments: polarization can be quickly restored to the maximal level within only 15 seconds with the result that polarization levels stay constant over several hundred experiments. The techniques outlined are applicable to hyper-polarizing spin-1/2 hetero-nuclei, such as ${ }^{13} \mathrm{C},{ }^{19} \mathrm{~F},{ }^{31} \mathrm{P}$, etc. Development of such methods opens new avenues in NMR spectroscopy and imaging, which were out of reach for sensitivity reasons.

Received 28th April 2015 Accepted 20th July 2015

DOI: $10.1039 / c 5 r a 13808 f$

www.rsc.org/advances hyperpolarization methods are getting more and more popular in NMR, since they enable ${ }^{1-4}$ new experiments in NMR spectroscopy and imaging. One of the widely used spin hyperpolarization methods is Para-Hydrogen Induced Polarization $(\mathrm{PHIP})^{5,6}$ and its recent off-spring Signal Amplification By Reversible Exchange (SABRE). ${ }^{7}$ In PHIP and SABRE, for sensitivity enhancement the spin order of parahydrogen $\left(\mathrm{p}-\mathrm{H}_{2}\right)$ is exploited. $\mathrm{p}-\mathrm{H}_{2}$ is the dihydrogen molecule in its nuclear singlet spin state, which can be relatively easily enriched at low temperature. In PHIP experiments, $\mathrm{p}-\mathrm{H}_{2}$ is chemically attached to a substrate, which is a molecule having a double or triple $\mathrm{C}-\mathrm{C}$ bond, in such a way that the two protons originating from $\mathrm{p}-\mathrm{H}_{2}$ occupy non-equivalent sites in the reaction product. In SABRE, chemical modification of the substrate is avoided: the substrate, $\mathrm{S}$, gets hyperpolarized in a transient complex $\left(\mathrm{cH}_{2}\right)-(\mathrm{Cat})-\mathrm{S}$ due to spin order transfer from parahydrogen (here Cat denotes a SABRE catalyst), see Scheme 1. Hereafter $c \mathrm{H}_{2}$ denotes $\mathrm{H}_{2}$ in the form of dihydride bound to the complex. Since the substrate is restored after leaving the complex, SABRE enables rehyperpolarizing its nuclear spins and retaining the NMR enhancement. Thus, SABRE is a very promising hyperpolarization method for novel experiments in NMR spectroscopy and imaging. ${ }^{7-9}$

A bottleneck of the SABRE method is the polarization transfer to the substrate; hence, optimizing the transfer efficiency is critical for the performance of the technique. It is 


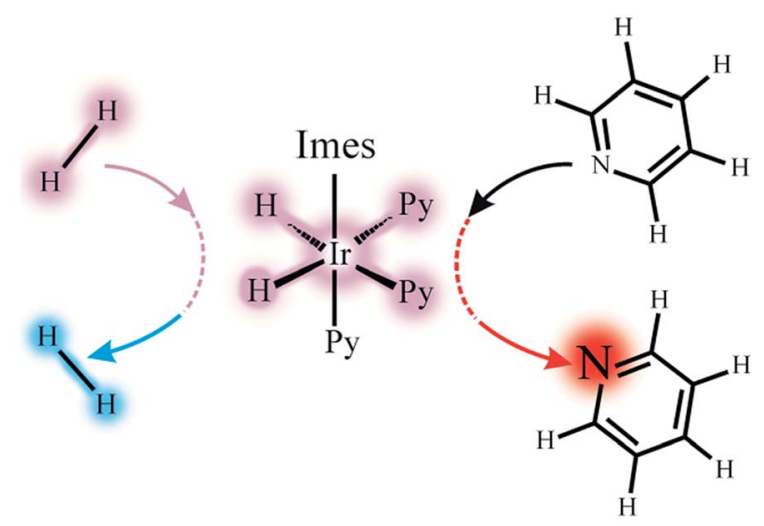

Scheme 1 Scheme of SABRE formation and polarization transfer to nitrogen. Parahydrogen and Py enter the SABRE complex (here the Irlmes complex is shown). After spin mixing $\mathrm{H}_{2}$ goes from the singlet (para) to a triplet (ortho) state, while mainly the ${ }^{15} \mathrm{~N}$-nuclei of the two equatorial pyridines acquire spin hyperpolarization. Also, the equatorial pyridine exchanges with free pyridine leading to transfer of hyperpolarization from the complex to the free substrate.

known that at low magnetic fields an efficient coherent process for polarization transfer is existing; its mechanism is wellestablished for proton-proton transfer. ${ }^{7,10-12}$ However, in many NMR experiments, it is crucial to enhance signals of "insensitive”, but important NMR hetero-nuclei, such as ${ }^{13} \mathrm{C}$ and ${ }^{15} \mathrm{~N}$. Such nuclei are often advantageous for NMR detection because of longer spin relaxation times (consequently, longer lifetimes of spin hyperpolarization) and lower background signals as compared to protons. In this work, we study hetero-nuclear polarization transfer in SABRE experiments and demonstrate enhancement of NMR signals of ${ }^{15} \mathrm{~N}$ as an example. Exploiting SABRE for such purposes is highly promising. Polarization transfer of SABRE-derived hyperpolarization to "insensitive" NMR has been demonstrated previously but not yet studied in full detail. It has been shown ${ }^{7,13,14}$ that SABRE enables hyperpolarization of ${ }^{13} \mathrm{C}$ and ${ }^{15} \mathrm{~N}$ nuclei at low magnetic fields. Very recently, Zhivonitko et al. ${ }^{15}$ have demonstrated polarization transfer to the ${ }^{31} \mathrm{P}$ nuclei, present in the SABRE complex, occurring at very low fields. Theis et $a l .{ }^{16}$ proposed a technique (LIGHT-SABRE) for polarization transfer at high fields based on special NMR pulse sequences. Here, to obtain the desired NMR signal enhancement we exploit different methods. First, we perform polarization transfer at ultralow magnetic fields and at low magnetic fields. Such methods avoid using NMR pulses and are based on coherent spin mixing under "strong coupling" conditions, vide infra. Then, we perform experiments based on RF-excitation at high fields based on a recently proposed polarization transfer method. ${ }^{17}$ This study significantly extends the capabilities of the SABRE method for enhancing weak signals of "insensitive" NMR nuclei because it avoids technically demanding field-cycling. High-field SABRE experiments can be performed at a constant field strength using standard NMR equipment. Furthermore, an important aspect of highfield SABRE, as demonstrated here, is that this method enables continuous production of hyperpolarization: within a few seconds the NMR signal enhancement is restored. The experiment can be repeated at least several hundred times without any loss of signal. Potential applications of our method are discussed in the paper.

\section{Methods}

\section{A. Sample preparation}

As SABRE substrates we used ${ }^{15} \mathrm{~N}$-pyridine $\left({ }^{15} \mathrm{~N}\right.$-Py) and as a SABRE pre-catalyst we used: IrImesCODCl (Imes $=1,3$-bis $(2,4,6$ -

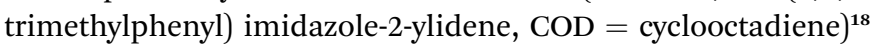
and Crabtree's catalyst, $\operatorname{IrP}\left(\mathrm{C}_{6} \mathrm{H}_{11}\right)_{3} \mathrm{PyCOD} \mathrm{PF}_{6}$ (ref. 13) (all experimental results for Crabtree's catalyst are in ESI $\dagger$ ). In our experiments we used a mixture of $70 \mathrm{mM}$ of ${ }^{15} \mathrm{~N}$-Py and $2 \mathrm{mM}$ of Ir pre-catalyst. Crabtree's catalyst was purchased from ABCR $\mathrm{GmbH}$, IrImesCODCl was synthesized using a procedure described before, ${ }^{19}$ methanol-d $\mathrm{d}_{4}$ and ${ }^{15} \mathrm{~N}$-Py were purchased from Deutero $\mathrm{GmbH}$; and all other compounds were purchased from Sigma-Aldrich. Samples were used without additional purification. Parahydrogen was prepared using a Bruker parahydrogen generator with an enrichment of $92 \%$ in $\mathrm{p}-\mathrm{H}_{2}$ and stored in a purified gas-balloon prior to the experiments.

To synthesize deuterated ${ }^{15} \mathrm{~N}-\mathrm{Py}, 2.2 \mathrm{~g}$ of ${ }^{15} \mathrm{~N}$-Py were heated with $\mathrm{D}_{2} \mathrm{O}(15 \mathrm{ml} 99.9 \%)$ and a mixture of $\mathrm{Pt} / \mathrm{Pd} /$ carbon $(1 \mathrm{~g} 10 \%$ $\mathrm{Pt} /$ carbon and $0.5 \mathrm{~g} 10 \% \mathrm{Pd} /$ carbon) in a closed high pressure vessel at $185{ }^{\circ} \mathrm{C}$ for 50 hours. After cooling, the deuterated ${ }^{15} \mathrm{~N}$-Py was salted out by addition of $10 \mathrm{~g} \mathrm{Na} \mathrm{SO}_{4}$. The two phases were separated and Py was distilled three times in a closed vacuum manifold system versus liquid nitrogen. The last distillation was done after Py was dried over molecular sieves of $4 \AA$ A pore diameter. The yield was $1.5 \mathrm{~g}$, the degree of deuteration was around $95 \%$.

\section{B. NMR experiments}

To assign lines in the SABRE spectra we performed additional NMR experiments. Proton signal assignments are known from previous works. ${ }^{13}$ In experiments with both catalysts three forms of Py are observed in the NMR spectrum, which correspond to free Py in solution (fPy) and to catalyst bound Py ligands in the equatorial (ePy) and axial (aPy) positions, see Scheme 1. Likewise, in the ${ }^{15} \mathrm{~N}$ spectra there are signals coming from all these three Py forms. To assign these signals we performed two-dimensional HSQC-NMR experiments on thermally polarized ${ }^{15} \mathrm{~N}-\mathrm{Py} .{ }^{20}$ In the resulting two-dimensional spectra cross-peaks appear only between protons and ${ }^{15} \mathrm{~N}$ nuclei belonging to the same Py form: this enabled unambiguous NMR signal assignment for nitrogen as well. The HSQC spectra and the signal assignment are presented in ESI. $\dagger$

\section{SABRE experiments}

SABRE experiments were done in the following way.

Low-field SABRE experiments. Here we bubbled the $\mathrm{p}-\mathrm{H}_{2}$ gas through the sample located either at a low magnetic field comparable to the Earth field or at a very low field inside a $\mu$-metal box. After bubbling, the sample was brought manually to the measurement position, i.e., to the high field of $9.4 \mathrm{~T}$, 
within a time period of about 5 seconds. At the homogeneous field of the NMR spectrometer, a detecting RF-pulse was applied and the NMR spectrum was obtained as the Fourier transform of the Free Induction Decay (FID) signal. NMR observation was done for both, protons and ${ }^{15} \mathrm{~N}$ nuclei.

High-field SABRE experiments. Here we did the bubbling inside the NMR spectrometer, i.e., directly at the observation field of $9.4 \mathrm{~T}$. After the bubbling we performed polarization transfer in the presence of two RF-fields resonant to protons $\left(\mathrm{RF}_{\mathrm{H}}\right.$-field) and to ${ }^{15} \mathrm{~N}$ nuclei $\left(\mathrm{RF}_{\mathrm{N}}\right.$-field), see Scheme 2 . The transfer scheme is the same as in our recent work: ${ }^{17}$ the $\mathrm{RF}_{\mathrm{H}}$-field is permanently turned on, whereas the $\mathrm{RF}_{\mathrm{N}}$-field is slowly (namely, in an adiabatic way) reduced from its initial value to zero. Here we used a linear profile for switching off the $\mathrm{RF}_{\mathrm{N}}$-field. The resulting polarization transferred to the heteronucleus is sensitive to the frequency and amplitude of both RF-fields, see below. The polarized NMR spectra of the nitrogen spins were obtained by applying a detecting pulse at the NMR frequency of ${ }^{15} \mathrm{~N}$ nuclei as the Fourier transform of the FID.

Bubbling method. The bubbling system was designed in such a way that one can create hyperpolarization using $\mathrm{p}-\mathrm{H}_{2}$ inside the NMR spectrometer without loss of field homogeneity. For that purpose we bubbled our sample in the following way: we fix a thin plastic capillary in a standard NMR tube so that it does not cause disturbance of homogeneity. Exploiting two electrically controlled gas valves we can automatically start and stop the bubbling: one valve opens the $\mathrm{p}-\mathrm{H}_{2}$ supply and the other allows us to release overpressure in the gas line to stop bubbling. Moreover, the plastic capillary is flexible enough to allow us to bubble the sample also outside the NMR-spectrometer and, after the end of the bubbling, to immediately transfer the sample to the detection position.

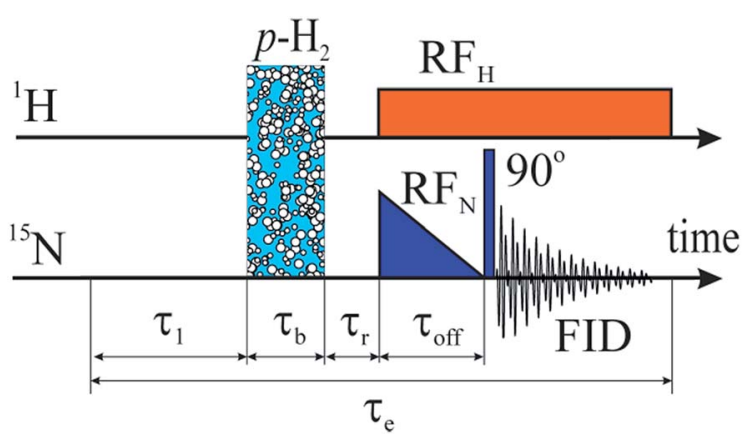

Scheme 2 Experimental protocol used for transfer of the SABREderived polarization to ${ }^{15} \mathrm{~N}$ nuclei. First, the spin system relaxes to thermal equilibrium during time period $\tau_{1}$. To generate spin hyperpolarization the $\mathrm{p}-\mathrm{H}_{2}$ gas is bubbled through the sample during the time period $\tau_{\mathrm{b}}$; it is necessary to wait a time period $\tau_{\mathrm{r}} \sim 0.5-1 \mathrm{~s}$ to remove all the bubbles from the sample. After that, the $\mathrm{RF}_{\mathrm{H}}$-field with constant amplitude is switched on in the proton channel while the $\mathrm{RF}_{\mathrm{N}}$-field is switched on in the ${ }^{15} \mathrm{~N}$ channel. The amplitude of the $\mathrm{RF}_{\mathrm{N}}$-field is linearly reduced to zero during the time $\tau_{\text {off }}$ and the free induction decay (FID) is measured after applying a 90 -degree pulse on nitrogens. The total time of one experiment is denoted as $\tau_{\mathrm{e}}$.

\section{Results and discussion}

Now let us present the results of SABRE transfer to ${ }^{15} \mathrm{~N}$ nuclei at low magnetic fields and also at high field in the presence of RF-excitation. In each case we exploit coherent re-distribution of polarization among "strongly coupled" spins (vide infra). When the "strong coupling" condition is violated and only cross-relaxation mechanisms are operative, i.e., at high magnetic field in the absence of RF-excitation, polarization transfer to the ${ }^{15} \mathrm{~N}$ nuclei becomes rather inefficient. This is demonstrated by Fig. 1: although at high field (but without RF-excitation) the nitrogen signals are enhanced as compared to the thermal signals, the enhancement factors are relatively small reaching approximately 10-20 for fPy and 130 for the Py ligands in the SABRE complex. These enhancements have been attributed to cross-relaxation mediated polarization transfer from $c \mathrm{H}_{2} \cdot{ }^{21}$ As we show below, enhancements resulting from coherent transfer are much larger.

The results are organized in three subsections. The low-field SABRE experiments logically split into two parts: experiments at ultralow field (in $\mu$-box) and experiments at the Earth field. The third subsection is dedicated to polarization transfer at high fields in the presence of resonant RF-fields.

\section{A. Polarization transfer at ultralow field}

Polarization transfer in the absence of RF-excitation can occur due to two mechanisms: stochastic (cross-relaxation) and coherent re-distribution of non-thermal spin order. Crossrelaxation is usually much slower and much less efficient as compared to the coherent mechanism. Thus, exploiting a

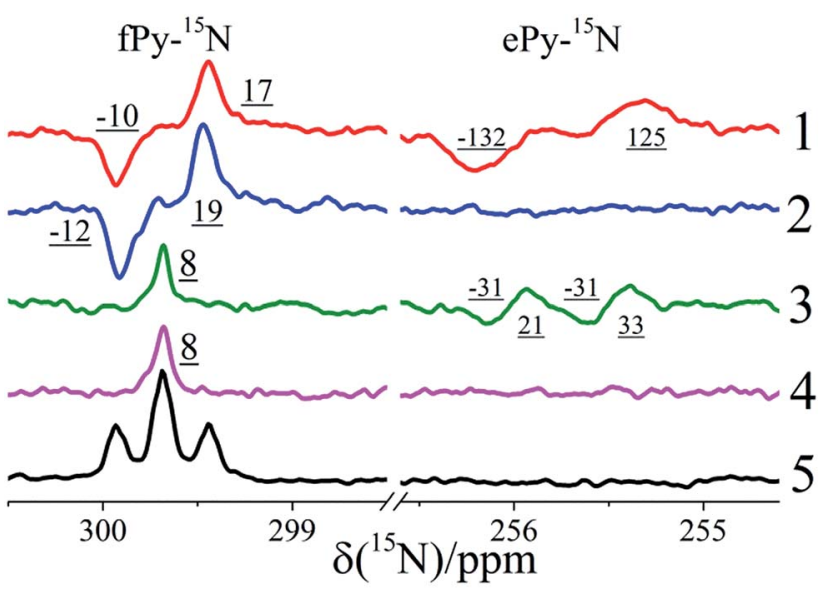

Fig. $1{ }^{15} \mathrm{~N}$ NMR spectrum the SABRE-derived polarization obtained at 9.4 T. Here the spectra are shown without proton decoupling (trace 1), with decoupling of $\mathrm{CH}_{2}$ (trace 2), with decoupling of the aromatic protons (trace 3) and with broadband ${ }^{1} \mathrm{H}$ decoupling (trace 4). The thermal NMR spectrum obtained after 5120 acquisitions (divided by a factor of 160, requires 43 hours of acquisition) is also shown for comparison (trace 5). The time of bubbling of $\mathrm{p}-\mathrm{H}_{2}$ is $30 \mathrm{~s}$; the delay between the end of the bubbling and measuring the spectra is $1 \mathrm{~s}$; the spectra were taken using a $\pi / 2$-pulse. Signal enhancements with respect to the thermal polarization are given by numbers for the lines in the fPy and ePy NMR multiplets. 
coherent transfer mechanism is advantageous for enhancing NMR signals of target spins; however, such mechanisms are known to be operative only at sufficiently low magnetic fields. Specifically, they require "strong coupling" of spins, meaning that the difference, $\delta \nu$, in the Zeeman interaction of spins with the external field is smaller than or comparable to their spinspin coupling, J. ${ }^{\mathbf{6 2 2 - 2 4}}$ The mechanism of such transfer is well understood; its coherent nature has been revealed by observing pronounced oscillations in the dependence of the transfer efficiency on the spin mixing time. For homo-nuclei, this condition can be fulfilled at relatively high fields;1,25,26 however, for hetero-nuclei, magnetic fields well below the Earth field are required. ${ }^{7,13-15,27,28}$ For instance, for ${ }^{1} \mathrm{H}$ and ${ }^{15} \mathrm{~N}$ nuclei having a scalar coupling of $J=10 \mathrm{~Hz}$ the strong coupling condition, $\delta \nu \leq$ $J$, is reached only at a field below $270 \mathrm{nT}$. To reach such low fields we placed the sample into a $\mu$-metal box and performed polarization transfer there.

The results are presented in Fig. 2: SABRE-enhanced NMR spectra of ${ }^{15} \mathrm{~N}$-Py are shown for nitrogen. We obtain significant enhancement for fPy, see the negatively polarized triplet with splitting due to interactions with the fPy protons (as follows from experiments with proton decoupling). Signal enhancements are also strong for $\mathrm{ePy}$, see the negatively polarized doublet with splitting due to interaction with both $c \mathrm{H}_{2}$ and the Py protons. Polarization of aPy is smaller but clearly visible. The NMR enhancements are about 2500 for fPy and ePy and about 200 for aPy enabling detection of nitrogen NMR in a single scan. The observed polarization is net polarization of purely emissive character: the enhanced lines do not vanish when proton decoupling is applied. For achieving a similar NMR signal (in

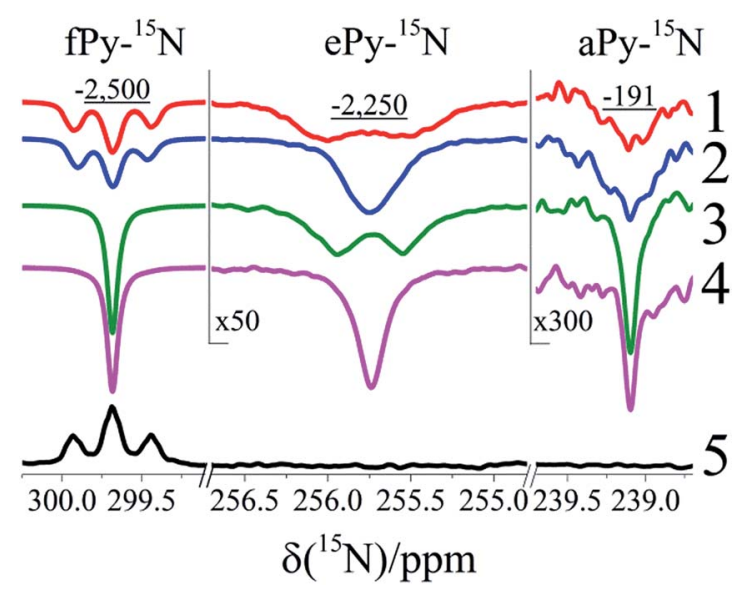

Fig. $2{ }^{15} \mathrm{~N}$ NMR spectra at $9.4 \mathrm{~T}$ showing SABRE-derived polarization after spin mixing in the $\mu$-metal box. Here the spectra are shown without proton decoupling (trace 1), with decoupling of $\mathrm{CH}_{2}$ (trace 2), with decoupling of the aromatic protons (trace 3) and with broadband ${ }^{1} \mathrm{H}$ decoupling (trace 4). The thermal NMR spectrum obtained after 5120 acquisitions (divided by a factor of 2 ) is also shown for comparison (trace 5). The time of bubbling $\mathrm{p}-\mathrm{H}_{2}$ is $30 \mathrm{~s}$; the delay between the end of the bubbling in the $\mu$-box and measuring the spectra is $6 \mathrm{~s}$; the spectra were taken using a $\pi / 2$-pulse. Signal enhancements (calculated for integrated signals corresponding to NMR multiplets) with respect to the thermal polarization are given by numbers for the NMR multiplets of fPy, ePy and aPy. terms of intensity, but not of signal-to-noise ratio) using thermal polarization it is necessary to make several thousand acquisitions.

As a direct evidence for strong coupling of the proton and nitrogen spins at very low fields one can take the fact that the lifetime of the nitrogen polarization in the $\mu$-metal box is considerably shorter than the ${ }^{15} \mathrm{~N} \mathrm{~T}_{1}$-relaxation time at high magnetic fields, where the hetero-nuclei are coupled weakly. The reduction of the relaxation time is approximately by an order of magnitude, see ESI: $\uparrow$ from $60 \mathrm{~s}$ at high field where spins are weakly coupled to $6 \mathrm{~s}$ at ultralow field. This observation agrees with results obtained by Truong et al. ${ }^{29}$ The dramatic reduction of the relaxation time is attributed to strong coupling of the ${ }^{15} \mathrm{~N}$ spin to protons: it is known that strongly coupled spins tend to relax with a common $\mathrm{T}_{1}$-relaxation time. ${ }^{30,31}$

Similar results can be obtained using another SABRE catalyst, Crabtree's catalyst. Typical enhancements are about 30-40 for ${ }^{15} \mathrm{~N}$ of fPy, which are demonstrated in ESI. $\dagger$

Enhancements obtained with protonated and deuterated ${ }^{15} \mathrm{~N}$ Py are close to each other, see Fig. 3. The only difference is that due to H-D exchange for the ortho-protons of Py in the SABRE complex (as reported previously in ref. 21) in experiments with the deuterated substrate three polarized species of ${ }^{15} \mathrm{~N}$-Py are present having slightly different chemical shifts, see Fig. 3. Signals of the two partly protonated ${ }^{15} \mathrm{~N}$-Py are affected by proton decoupling in contrast to that of the fully deuterated substrate.

Thus, SABRE experiments exploiting spin mixing at ultralow field enable strong polarization of both proton and ${ }^{15} \mathrm{~N}$ spins of

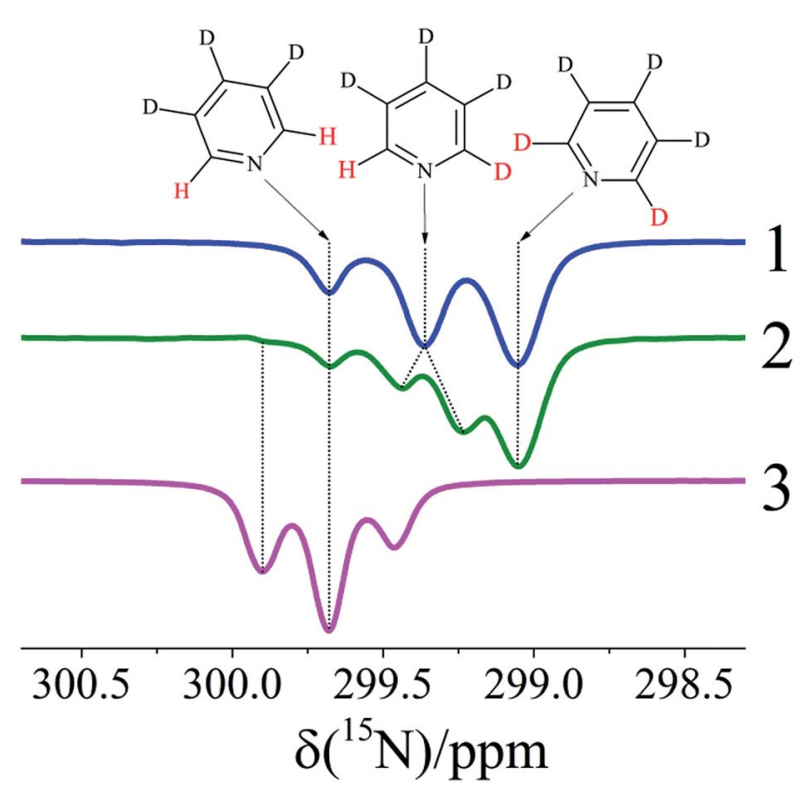

Fig. $3{ }^{15} \mathrm{~N}$ NMR spectra at $9.4 \mathrm{~T}$ showing SABRE-derived polarization after spin mixing in the $\mu$-metal box. Here traces 1 and 2 show the spectra obtained with perdeuterated $P y-D_{5}$ with (1) and without (2) proton decoupling. Vertical dotted lines mark the ${ }^{15} \mathrm{~N}$ NMR signals originating from the starting $P y-D_{5}$, and pyridine molecules with one and two protons in the ortho position that are formed by isotope exchange in the Irlmes complex. Experimental conditions are the same as those for spectra shown in Fig. 2. The spectrum obtained with protonated Py is shown by trace 3 . 
the Py molecule. The enhancements achieved in this way reach an impressive value of 2500 for a fPy concentration of about 64 $\mathrm{mM}$ that is close to the results obtained by Theis et al. ${ }^{14}$ when a similar mixture is used.

\section{B. Polarization transfer at the Earth field}

Running experiments at ultralow fields can often be demanding and technically difficult. In such a situation a remedy is performing the SABRE experiment at a relatively low field, which is stronger than or comparable to the Earth field. In this case it is not possible to form net magnetization of the ${ }^{15} \mathrm{~N}$ spins: indeed, simultaneous flips and flops of the protons and nitrogen spins become forbidden as they require an energy much larger than $J$. Consequently, scalar spin-spin couplings are not sufficient for such a polarization transfer; hence, net polarization of the ${ }^{15} \mathrm{~N}$ spins cannot be formed by the coherent mechanism based on "strong coupling". However, it is possible to form multiplet (or anti-phase) polarization of the proton and nitrogen spins. The requirements are then that (i) the proton spin system is strongly coupled and (ii) different protons have unequal couplings to the hetero-nucleus. In this situation the coherent transfer mechanism is operative again and multiplet polarization of protons and hetero-nuclei can be formed. ${ }^{32,33}$ In this case polarization integrated over all NMR lines of the hetero-nucleus is zero, but individual lines can be strongly enhanced.

Here, we demonstrate the corresponding effect in SABRE by performing spin mixing and polarization transfer at low fields, specifically, at the Earth field. The results for SABRE after such a preparation of polarization at the Earth field are shown in Fig. 4. It is readily seen that fPy has no net polarization, neither for protons nor for nitrogen. For instance, the nitrogen polarization vanishes when proton decoupling is used. However, individual lines of NMR multiplets for both magnetic nuclei are strongly enhanced. The absolute enhancement of the lines is about 1600, similar to that achieved after spin mixing at ultra-low field. This is an indication that in both cases spin mixing is equally efficient and polarization transfer relies on a coherent mechanism. Similar ${ }^{15} \mathrm{~N}$ polarization patterns have been obtained by Atkinson et al. ${ }^{13}$ and by Truong et al.; ${ }^{29}$ in the latter work multiplet polarization on ${ }^{13} \mathrm{C}$ nuclei has also been reported.

Each of the methods, polarization at low and ultralow magnetic field, has its advantages. At ultralow fields it is possible to net polarize the nitrogen spins (net polarization is usually easier to deal with in NMR spectroscopy and imaging), whereas performing polarization transfer at the Earth field is easier from the technical point of view. Corresponding ${ }^{1} \mathrm{H}$ spectra are shown in ESI $\uparrow$ together with the results obtained with Crabtree's catalyst.

For practical reasons, it is preferable to develop polarization transfer schemes being efficient at high fields, where modern NMR spectrometers usually operate. In this situation the "strong coupling" condition is obviously violated, since the mechanism described above is operative exclusively at low-fields. Nonetheless, it is possible to fulfil the conditions of strong coupling (i.e., to mimic the low-field situation) at high

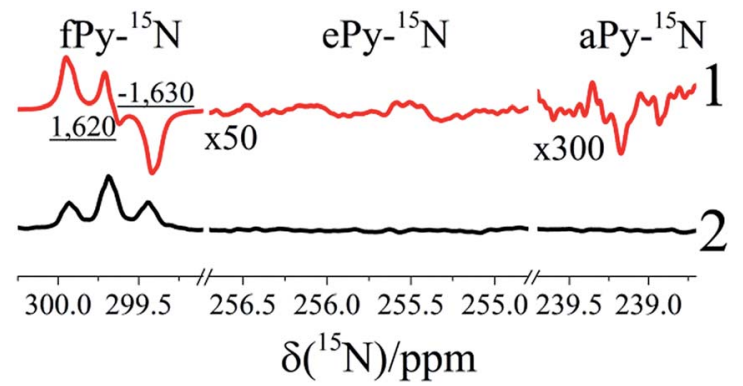

Fig. $4{ }^{15} \mathrm{~N}$ NMR spectra at 9.4 T showing SABRE-derived polarization after spin mixing at the Earth magnetic field (trace 1); the thermal NMR spectrum obtained after 5120 acquisitions (divided by a factor of 2) is also shown for comparison (trace 2). The time of bubbling $\mathrm{p}-\mathrm{H}_{2}$ is $30 \mathrm{~s}$; the delay between the end of the bubbling in the $\mu$-box and measuring the spectra is $6 \mathrm{~s}$; both spectra were taken using a $\pi / 2$-pulse. Signal enhancements with respect to the thermal polarization are given by numbers for lines in the fPy NMR multiplet. There is no significant polarization of Py in the complex.

fields by performing resonant RF-excitation of the spin system. As has been shown previously, when the difference of the precession frequencies of spins about their effective fields in the rotating frame is smaller than or comparable to $J$ coherent polarization transfer becomes operative again. In this way, originally low-field experiments can be performed at high field as has been demonstrated, for instance, for proton SABRE experiments. $^{34}$ Here, we pursue the same strategy as we described before ${ }^{\mathbf{1 7}}$ to polarize the nitrogen spins at high field in order to avoid the necessity of field variation.

\section{Polarization transfer at high field}

SABRE experiments at high field were done in accordance with the experimental protocol depicted in Scheme 2. We systematically varied the frequency, $\nu_{\mathrm{rf}}^{\mathrm{N}}$, of the $\mathrm{RF}_{\mathrm{N}}$-field; the $\mathrm{RF}_{\mathrm{H}}$-frequency, $\nu_{\mathrm{rf}}^{\mathrm{H}}$, was always set resonant to the NMR signals of $c \mathrm{H}_{2}$. We always used the same $\mathrm{RF}_{\mathrm{H}}$-field amplitude, $\nu_{1}^{\mathrm{H}}=$ $75 \mathrm{~Hz}$; the starting $\mathrm{RF}_{\mathrm{N}}$-field amplitude, $\nu_{1}^{\mathrm{N}}$, was set to the slightly higher value of $80 \mathrm{~Hz}$. We deliberately set the RF-amplitudes low: at such settings the RF power exerted on the sample is kept low and the two RF-fields do not heat the sample, which is an advantage of our method. The low RF-excitation bandwidth, however, also brings about a disadvantage of the method: one cannot transfer polarization simultaneously over a wide range of chemical shifts. At low $\nu_{1}^{\mathrm{N}}$ such a transfer can only be achieved by varying the $\nu_{\mathrm{rf}}^{\mathrm{N}}$ frequency. Subsequently, the $\mathrm{RF}_{\mathrm{N}}$-field was reduced in such a way that the spin system passed through a Level Anti-Crossing (LAC). At this LAC the protonnitrogen system becomes "strongly coupled" and coherent spin mixing is operative. The LAC condition implies that $\nu_{\text {eff }}^{\mathrm{N}} \approx$ $\nu_{\mathrm{eff}}^{\mathrm{H}}$, i.e., the frequencies of spin precession about the corresponding effective fields in the rotating frame should be almost equal. ${ }^{17}$ Each effective field is the vector sum of the transverse RF-field and the residual field along the magnetic field axis in the rotating frame (the reference frame rotating with the RF-field). When the proton-proton coupling, $J_{\mathrm{HH}}$, is the largest 
coupling in the system the LAC condition is given by $\left(\nu_{\text {eff }}^{\mathrm{H}}-\nu_{\text {eff }}^{\mathrm{N}}\right)=J_{\mathrm{HH}} \cdot{ }^{17}$ As we have shown in a previous study on analogous PHIP experiments the polarization transfer method works for arbitrary relation between the proton-proton and proton-nitrogen couplings; the only prerequisite is that the $\mathrm{RF}_{\mathrm{N}}$-field is switched off slowly enough to guarantee adiabatic passage through the relevant LACs; details are given in ref. 17. In general, LACs are known to provide efficient polarization transfer pathways in NMR. ${ }^{22,35-37}$ Likewise, NMR methods based on adiabatic polarization transfer are well-established since the pioneering work by Chingas et $a l .{ }^{37}$ and later works; ${ }^{38-45}$ in the present work we have chosen the method ${ }^{17}$ optimized for transferring the singlet spin order to net spin magnetization.

The transfer of such a SABRE-derived polarization to ${ }^{15} \mathrm{~N}$ nuclei is demonstrated in Fig. 5 . It is seen that nitrogen can be strongly polarized; typical enhancements are about \pm 800 for fPy and \pm 12000 for $\mathrm{ePy}$. Interestingly, by varying the $\mathrm{RF}_{\mathrm{N}}$-frequency it is possible to change the polarization phase: the enhanced ${ }^{15} \mathrm{~N}$ Py signals are emissive or absorptive depending on $\nu_{\mathrm{rf}}^{\mathrm{N}}$. This is in contrast to the low-field experiments where the polarization phase is dictated by the spin dynamics in the SABRE complex: since only one parameter, the external static field, can be varied one cannot manipulate the polarization phase. In experiments with RF-excitation there are two variable parameters: $\mathrm{RF}_{\mathrm{N}}$-field frequency and strength, thus providing more flexibility in manipulating the spin polarization. Details of the corresponding spin dynamics have been explained in detail in our previous publication. ${ }^{17}$ Thus, the proposed method for transferring PHIP to polarization of hetero-nuclei is applicable to SABRE despite the fact that the coupled proton-nitrogen spin system is formed only transiently, i.e., the proton-nitrogen couplings responsible for polarization transfer are switched on and off in the course of the transfer process. Nevertheless, polarization transfer is efficient resulting in ${ }^{15} \mathrm{~N}$ NMR signal enhancements of about 800 for fPy; for ePy having weaker thermal NMR signal the signal enhancement is about 12000 . Analogous results can be obtained using Crabtree's catalyst, see ESI; $†$ typical enhancement values are about 900 for ePy.

\section{Continuous production of hyperpolarization}

An attractive peculiarity of the SABRE method is that it allows one to continuously re-hyperpolarize spins: as shown by Hövener et al. ${ }^{46}$ under constant supply of parahydrogen the substrate spins quickly restore their SABRE-derived NMR enhancement after it is destroyed in the detection process. This is in contrast to many other hyperpolarization methods in liquids. Techniques utilizing chemical reactions to form hyperpolarization, for instance, the traditional PHIP method and the CIDNP method, ${ }^{47}$ are usually subject to sample depletion after several experiments. Dissolution DNP, ${ }^{48}$ which provides impressive NMR enhancements of 10000 or more is limited to single-shot experiments and slow formation of hyperpolarization. Thus, these methods are usually incompatible with experiments, which require signal accumulation. Here, we demonstrate that high-field SABRE is free of all these
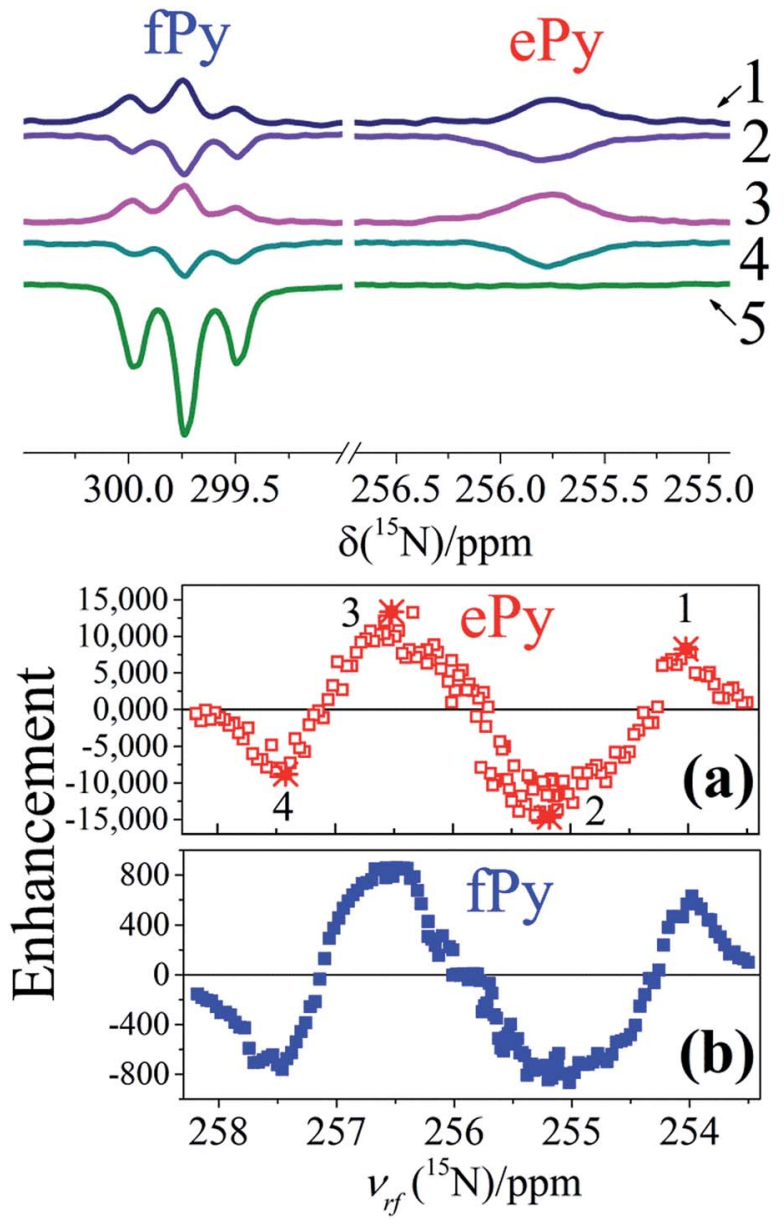

Fig. 5 Transfer of SABRE-derived polarization from protons to ${ }^{15} \mathrm{~N}$ spins at high magnetic field of 9.4 Tesla. The upper plot shows the ${ }^{15} \mathrm{~N}$ NMR spectra obtained using the protocol shown in Scheme 2. Here $v_{1}^{H}=75 \mathrm{~Hz}, v_{\mathrm{rf}}^{\mathrm{H}}=-22.8 \mathrm{ppm}, v_{1}^{\mathrm{N}}=80 \mathrm{~Hz}, \tau_{\text {off }}=0.5 \mathrm{~s}$ and $\nu_{\mathrm{rf}}^{\mathrm{N}}=254 \mathrm{ppm}$ (trace 1), $255.2 \mathrm{ppm}$ (trace 2), $256.5 \mathrm{ppm}$ (trace 3) and $257.4 \mathrm{ppm}$ (trace 4); the spectrum obtained after spin mixing at ultralow field is shown for comparison (trace 5). The spectra were taken using a $\pi / 2$-pulse. The lower plot shows the dependence of the ${ }^{15} \mathrm{~N}$ signal enhancement for ePy (a) and fPy (b) on the $\mathrm{RF}_{\mathrm{N}}$-frequency; the stars and numbers indicate the $\nu_{\mathrm{rf}}^{\mathrm{N}}$ frequencies, for which the NMR spectra in the upper plot are shown.

drawbacks: polarization can be rapidly restored; the level of polarization stays constant over multiple measurements.

To demonstrate the possibility of re-hyperpolarizing spins we performed the following experiments. We utilized the method of polarization transfer shown in Scheme 2 and continuously repeated the experiment varying the time $\tau_{1}$ and therefore the total time of the experiment $\tau_{\mathrm{e}}$. The bubbling time $\tau_{\mathrm{b}}$ was $10 \mathrm{~s}$. We have run 5 subsequent series of measurements on the same sample with the following $\tau_{\mathrm{e}}: 40 \mathrm{~s}, 25 \mathrm{~s}, 15 \mathrm{~s}, 25 \mathrm{~s}$, $40 \mathrm{~s}$. The results are shown in Fig. 6. It is clearly seen that for each $\tau_{\mathrm{e}}$ value the signal goes to a constant level after several experiments. Moreover, the signal level is the same for the first experiment and the last experiment (in both cases $\tau_{\mathrm{e}}$ is the same). Thus, after approximately 300 measurements the signal stays constant. It is important to emphasize that the signal 
intensity depends on $\tau_{\mathrm{e}}$. This observation can be explained by two factors. First, shorter $\tau_{\mathrm{e}}$ means bigger average inflow of parahydrogen; consequently, at short $\tau_{\mathrm{e}}$ at the beginning of a cycle of the high-field SABRE experiment there is a higher concentration of residual parahydrogen from the previous cycle in the solution resulting in more efficient SABRE formation. Second, at longer $\tau_{\mathrm{e}}$ capillary effects become important: a certain amount of the polarized solution is lifted in the capillary, thus leaving the observation volume, therefore in experiments with different $\tau_{\mathrm{e}}$ the delivered amount of $\mathrm{p}-\mathrm{H}_{2}$ is different. Hence, for continuous high-field SABRE experiments it is optimal to use short $\tau_{\mathrm{e}}$ times. When the experimental timing is optimized, the NMR enhancement reaches 1600 for the fPy and approximately 25000 for ePy. The achieved results for fPy are comparable with ultralow field SABRE and for ePy are even better by an order of magnitude (compare Fig. 2 and 6).

Using the RF-pulse sequence one can create polarization only for complexes that contain substrate and singlet $c \mathrm{H}_{2}$. Moreover, the complex should be stable from the beginning of the pulse sequence until the spin-system is adiabatically brought through the LAC region. Hence, the maximum number of polarized molecules of the substrate is equal to the number of Ir complexes multiplied by a factor of 2 for the IrImes complex because polarization is transferred to the protons of two ePy. This is in contrast to the low-field SABRE case: at low fields polarization transfer is constantly occurring during repeated association-dissociation of the substrate. For this reason the ratio of polarization enhancement between fPy and ePy is different in RF-SABRE and $\mu$-box SABRE: at ultralow fields all polarized Py ligands undergo exchange and contribute to

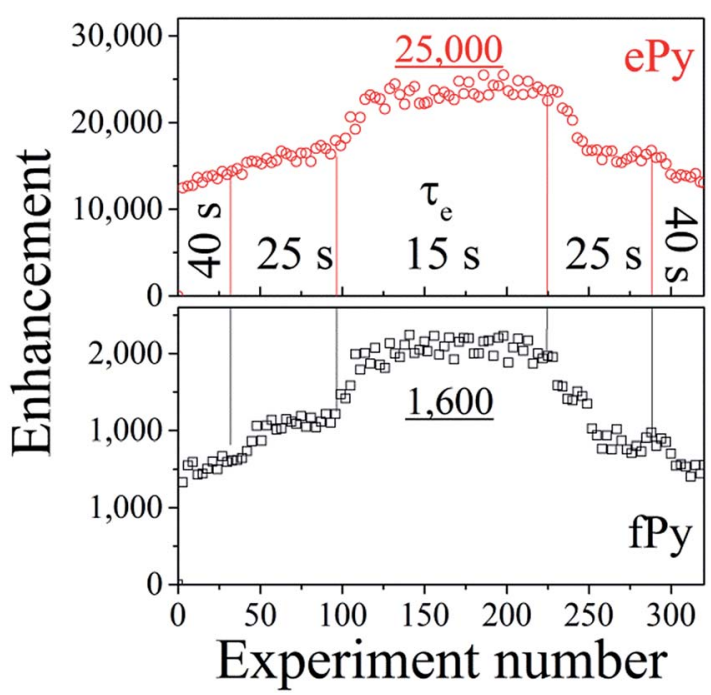

Fig. 6 Continuous re-hyperpolarization at high magnetic field, $B_{0}=$ 9.4 T, using the SABRE technique. In this experiment we repeated the experimental protocol shown in Scheme 2 varying the time period $\tau_{\mathrm{e}}$. Five consecutive series of measurements have been performed using the following $\tau_{\mathrm{e}}$ values: $40 \mathrm{~s}, 25 \mathrm{~s}, 15 \mathrm{~s}, 25 \mathrm{~s}, 40 \mathrm{~s}$. The NMR enhancement is shown as a function of the number of experiment for ePy (top) and fPy (bottom); the maximal achieved enhancements are 25000 and 1600, respectively. Here $v_{1}^{\mathrm{H}}=75 \mathrm{~Hz}, v_{\mathrm{rf}}^{\mathrm{H}}=-22.8 \mathrm{ppm}, v_{1}^{\mathrm{N}}=$ $80 \mathrm{~Hz}, \tau_{\text {off }}=0.5 \mathrm{~s}$ and $\nu_{\mathrm{rf}}^{\mathrm{N}}=254 \mathrm{ppm}$. polarization of fPy, whereas at high fields only SABRE complexes bound during the time until the LAC is passed produce polarization of fPy.

\section{Conclusions}

In this work, we demonstrate efficient transfer of SABRE-derived proton polarization to ${ }^{15} \mathrm{~N}$ nuclei. For optimizing the transfer process we exploited different possibilities: polarization transfer at low and ultralow external magnetic field as well as polarization transfer at high field in the presence of RF-excitation. Low-field polarization transfer is relatively easy to perform: it is only required to bring the system to a sufficiently low field to perform spin mixing among "strongly coupled" spins. The highest signals are achieved when the spin system is kept at an ultralow field, which can be achieved by using a $\mu$-box. In this situation, the resulting nitrogen polarization is of pure net character, thus, there is no cancellation of NMR signals with opposite phases. Polarization obtained after mixing at the Earth magnetic field is of multiplet character, i.e., in the NMR spectrum there are positive and negative lines, which cancel each other in the integral. However, this is not a problem when spectral resolution is sufficiently high; signal enhancement for individual lines is similar to that in the case of spin mixing at ultra-low field.

Furthermore, we have achieved strong enhancements for ${ }^{15} \mathrm{~N}$ spins at high field in the presence of RF-excitation at two frequencies using a special scheme for spin order transfer based on adiabatic passage through LACs in the rotating frame. When the LAC conditions are fulfilled the spin system becomes "strongly coupled" with the consequence that coherent polarization transfer becomes operative. With this scheme, enhancements of about 1000 are feasible. The method has further advantages: it enables manipulating the phase of the resulting nitrogen polarization and it is operative even when the RF-power is low. In the low-field experiments we achieved signal enhancement similar to that reported by Theis et al. ${ }^{\mathbf{1 6}}$ (when similar concentrations of substrates and catalysts are taken). In the high-field case we obtained an enhancement of about 1600 for free pyridine, a value that is almost 10 times higher than that reported by Theis et al. ${ }^{16}$ Our method has an additional advantage: in the case of LIGHT-SABRE ${ }^{\mathbf{1 6}}$ it is required to repeat the polarization transfer pulse sequence several times. The highest enhancement was achieved when the time period of the LIGHT-SABRE sequence was 7.5 seconds. In our case the polarization transfer pulse sequence lasts only $0.5 \mathrm{~s}$ allowing us to measure significant polarization on free pyridine and also on the complex bound substrate.

An important feature of the high-field SABRE technique demonstrated here is the possibility to re-hyperpolarize the substrate. Within only $15 \mathrm{~s}$ hyperpolarization is restored; repetitive measurements have not revealed any loss of signal over several hundred cycles of re-hyperpolarization. This enables continuous measurements with SABRE, which is advantageous for experiments that need accumulation.

Thus, we have demonstrated significant NMR enhancements for insensitive but important NMR nuclei. Achievable signal 
enhancements are about 2000 for free pyridine and over 25000 for pyridine in the SABRE complex. This enables numerous promising NMR experiments, which are currently not feasible for sensitivity reasons. We believe that the methods used in this work are general and can be used in other PHIP and SABRE experiments as well. Moreover, other spin-1/2 hetero-nuclei, such as ${ }^{13} \mathrm{C},{ }^{19} \mathrm{~F}$ and ${ }^{31} \mathrm{P}$ can be polarized by applying these methods thus paving the way to new applications of NMR spectroscopy and imaging.

\section{Acknowledgements}

This work was supported by the Russian Science Foundation (Grant No. 14-13-01053). H.M.V. acknowledges support by the Alexander von Humboldt Foundation. We acknowledge Dr Pavel Petrov for providing us with the IrImesCODCl pre-catalyst.

\section{Notes and references}

1 G. Pileio, S. Bowen, C. Laustsen, M. C. D. Tayler, J. T. HillCousins, L. J. Brown, R. C. D. Brown, J. H. ArdenkjaerLarsen and M. H. Levitt, J. Am. Chem. Soc., 2013, 135, 5084-5088.

2 C. Griesinger, M. Bennati, H.-M. Vieth, C. Luchinat, G. Parigi, P. Hofer, F. Engelke, S. J. Glaser, V. Denysenkov and T. F. Prisner, Prog. Nucl. Magn. Reson. Spectrosc., 2012, 64, 4-28.

3 K. V. Kovtunov, M. L. Truong, D. A. Barskiy, I. V. Koptyug, A. M. Coffey, K. W. Waddell and E. Y. Chekmenev, Chem.Eur. J., 2014, 20, 14629-14632.

4 S. Glöggler, J. Colell and S. Appelt, J. Magn. Reson., 2013, 235, 130-142.

5 S. B. Duckett and R. E. Mewis, Acc. Chem. Res., 2012, 45, 1247-1257.

6 J. Natterer and J. Bargon, Prog. Nucl. Magn. Reson. Spectrosc., 1997, 31, 293-315.

7 R. W. Adams, J. A. Aguilar, K. D. Atkinson, M. J. Cowley, P. I. P. Elliott, S. B. Duckett, G. G. R. Green, I. G. Khazal, J. López-Serrano and D. C. Williamson, Science, 2009, 323, 1708-1711.

8 N. Eshuis, B. J. A. van Weerdenburg, M. C. Feiters, F. P. J. T. Rutjes, S. S. Wijmenga and M. Tessari, Angew. Chem., Int. Ed., 2015, 54, 1481-1484.

9 N. Eshuis, N. Hermkens, B. J. A. van Weerdenburg, M. C. Feiters, F. P. J. T. Rutjes, S. S. Wijmenga and M. Tessari, J. Am. Chem. Soc., 2014, 136, 2695-2698.

10 H. Zeng, J. Xu, J. Gillen, M. T. McMahon, D. Artemov, J.-M. Tyburn, J. A. B. Lohman, R. E. Mewis, K. D. Atkinson, G. G. R. Green, S. B. Duckett and P. C. M. van Zijl, J. Magn. Reson., 2013, 237, 73-78.

11 A. N. Pravdivtsev, A. V. Yurkovskaya, H.-M. Vieth, K. L. Ivanov and R. Kaptein, ChemPhysChem, 2013, 14, 3327-3331.

12 E. B. Dücker, L. T. Kuhn, K. Münnemann and C. Griesinger, J. Magn. Reson., 2012, 214, 159-165.

13 K. D. Atkinson, M. J. Cowley, P. I. P. Elliott, S. B. Duckett, G. G. R. Green, J. López-Serrano and A. C. Whitwood, J. Am. Chem. Soc., 2009, 131, 13362-13368.
14 T. Theis, M. L. Truong, A. M. Coffey, R. V. Shchepin, K. W. Waddell, F. Shi, B. M. Goodson, W. S. Warren and E. Y. Chekmenev, J. Am. Chem. Soc., 2015, 137, 1404-1407.

15 V. V. Zhivonitko, I. V. Skovpin and I. V. Koptyug, Chem. Commun., 2015, 51, 2506-2509.

16 T. Theis, M. Truong, A. M. Coffey, E. Y. Chekmenev and W. S. Warren, J. Magn. Reson., 2014, 248, 23-26.

17 A. N. Pravdivtsev, A. V. Yurkovskaya, N. N. Lukzen, K. L. Ivanov and H. M. Vieth, J. Phys. Chem. Lett., 2014, 5, 3421-3426.

18 M. J. Cowley, R. W. Adams, K. D. Atkinson, M. C. R. Cockett, S. B. Duckett, G. G. R. Green, J. A. B. Lohman, R. Kerssebaum, D. Kilgour and R. E. Mewis, J. Am. Chem. Soc., 2011, 133, 6134-6137.

19 I. Kownacki, M. Kubicki, K. Szubert and B. Marciniec, J. Organomet. Chem., 2008, 693, 321-328.

20 G. Bodenhausen and D. J. Ruben, Chem. Phys. Lett., 1980, 69, 185-189.

21 D. A. Barskiy, K. V. Kovtunov, I. V. Koptyug, P. He, K. A. Groome, Q. A. Best, F. Shi, B. M. Goodson, R. V. Shchepin, A. M. Coffey, K. W. Waddell and E. Y. Chekmenev, J. Am. Chem. Soc., 2014, 136, 3322-3325.

22 K. L. Ivanov, A. N. Pravdivtsev, A. V. Yurkovskaya, H.-M. Vieth and R. Kaptein, Prog. Nucl. Magn. Reson. Spectrosc., 2014, 81, 1-36.

23 R. A. Green, R. W. Adams, S. B. Duckett, R. E. Mewis, D. C. Williamson and G. G. R. Green, Prog. Nucl. Magn. Reson. Spectrosc., 2012, 67, 1-48.

24 S. E. Korchak, K. L. Ivanov, A. V. Yurkovskaya and H.-M. Vieth, Phys. Chem. Chem. Phys., 2009, 11, 11146-11156.

25 L. Buljubasich, M. B. Franzoni, H. W. Spiess and K. Münnemann, J. Magn. Reson., 2012, 219, 33-40.

26 A. N. Pravdivtsev, A. V. Yurkovskaya, R. Kaptein, K. Miesel, H.-M. Vieth and K. L. Ivanov, Phys. Chem. Chem. Phys., 2013, 15, 14660-14669.

27 H. Johannesson, O. Axelsson and M. Karlsson, C. R. Phys., 2004, 5, 315-324.

28 T. Theis, P. Ganssle, G. Kervern, S. Knappe, J. Kitching, M. P. Ledbetter, D. Budker and A. Pines, Nat. Phys., 2011, 7, 571-575.

29 M. L. Truong, T. Theis, A. M. Coffey, R. V. Shchepin, K. W. Waddell, F. Shi, B. M. Goodson, W. S. Warren and E. Y. Chekmenev, J. Phys. Chem. C, 2015, 119, 8786-8797.

30 K. Ivanov, A. Yurkovskaya and H.-M. Vieth, J. Chem. Phys., 2008, 129, 234513.

31 A. N. Pravdivtsev, A. V. Yurkovskaya, H.-M. Vieth and K. L. Ivanov, J. Chem. Phys., 2014, 141, 155101.

32 M. Plaumann, U. Bommerich, T. Trantzschel, D. Lego, S. Dillenberger, G. Sauer, J. Bargon, G. Buntkowsky and J. Bernarding, Chem.-Eur. J., 2013, 19, 6334-6339.

33 L. T. Kuhn and J. Bargon, Top. Curr. Chem., 2007, 276, 25-68. 34 A. N. Pravdivtsev, A. V. Yurkovskaya, H. M. Vieth and

K. L. Ivanov, Phys. Chem. Chem. Phys., 2014, 16, 24672-24675. 35 D. T. Edmonds, Phys. Rep., 1977, 29, 234-290.

36 J. M. Millar, A. M. Thayer, A. Bielecki, D. B. Zax and A. Pines, J. Chem. Phys., 1985, 83, 934-938. 
37 G. C. Chingas, A. N. Garroway, W. B. Moniz and R. D. Bertrand, J. Am. Chem. Soc., 1980, 102, 2526-2528.

38 S. Hediger, B. H. Meier, N. D. Kurur, G. Bodenhausen and R. R. Ernst, Chem. Phys. Lett., 1994, 223, 283-288.

39 P. Pelupessy and E. Chiarparin, Concepts Magn. Reson., 2000, 12, 103-124.

40 P. Hodgkinson and A. Pines, J. Chem. Phys., 1997, 107, 87428751.

41 M. H. Levitt, J. Chem. Phys., 1991, 94, 30-38.

42 P. Hodgkinson, C. Auger and L. Emsley, J. Chem. Phys., 1998, 109, 1873-1884.

43 T. R. Eykyn, F. Ferrage, E. Winterfors and G. Bodenhausen, ChemPhysChem, 2000, 1, 217-221.

44 N. D. Kurur and G. Bodenhausen, J. Magn. Reson., Ser. A, 1995, 114, 163-173.
45 G. Zandomeneghi and B. H. Meier, J. Biomol. NMR, 2004, 30, 303-309.

46 J. B. Hövener, N. Schwaderlapp, T. Lickert, S. B. Duckett, R. E. Mewis, L. A. R. Highton, S. M. Kenny, G. G. R. Green, D. Leibfritz, J. G. Korvink, J. Hennig and D. von Elverfeldt, Nat. Commun., 2013, 4, 2946.

47 K. M. Salikhov, Y. N. Molin, R. Z. Sagdeev and A. L. Buchachenko, Spin polarization and magnetic effects in chemical reactions, Elsevier, Amsterdam, 1984.

48 J. H. Ardenkjaer-Larsen, B. Fridlund, A. Gram, G. Hansson, L. Hansson, M. H. Lerche, R. Servin, M. Thaning and K. Golman, Proc. Natl. Acad. Sci. U. S. A., 2003, 100, 1015810163. 